\title{
The Contribution of Dysfunctional Chloride Channels to Neurovascular Deficiency and Neurodegeneration
}

\author{
David A. Gascoigne ${ }^{1}$, Alexander Drobyshevsky ${ }^{2}$ and Daniil P. Aksenov ${ }^{1,3 *}$ \\ ${ }^{1}$ Department of Radiology, NorthShore University HealthSystem, Evanston, IL, United States, ${ }^{2}$ Department of Pediatrics, \\ NorthShore University HealthSystem, Evanston, IL, United States, ${ }^{3}$ Department of Anesthesiology, NorthShore University \\ HealthSystem, Evanston, IL, United States
}

Keywords: GABA, hypoxia, development, anesthesia, interneurons

\section{INTRODUCTION}

The brain is a metabolically demanding organ and its health directly depends on maintaining tissue oxygen that is sufficiently high to prevent hypoxia. Focal increases in oxygen demand, in response to sensory signals, motor output, etc., are supported by transient increases in cerebral blood flow via the hemodynamic response (Aksenov et al., 2016). Traditionally, specific products of glutamatergic and astrocytic pathways (i.e., nitric oxide (NO), arachidonic acid metabolites, calcium $\left(\mathrm{Ca}^{2+}\right)$ and potassium $\left(\mathrm{K}^{+}\right)$ions) have been proposed as mechanistic contributors to the hemodynamic response (Archer et al., 1994; Attwell et al., 2010; Ross, 2012; Nippert et al., 2018). However,

\section{OPEN ACCESS}

Edited by: Jacques Joubert, University of the Western Cape, South Africa

Reviewed by: Ana Margarida Ledo, University of Coimbra, Portugal

${ }^{*}$ Correspondence:

Daniil P. Aksenov daksenov@northshore.org

Specialty section: This article was submitted to Pharmacology of lon Channels and

Channelopathies,

a section of the journal

Frontiers in Pharmacology

Received: 06 August 2021 Accepted: 21 September 2021 Published: 04 October 2021

Citation: Gascoigne DA, Drobyshevsky A and Aksenov DP (2021) The Contribution of

Dysfunctional Chloride Channels to Neurovascular Deficiency and Neurodegeneration.

Front. Pharmacol. 12:754743. doi: 10.3389/fphar.2021.754743 these mechanisms may not be sufficient drivers of the hemodynamic response. For example, a recent review (Nippert et al., 2018) concluded that, although NO must be present for vasodilation to occur in the cerebral cortex, it is not the active signaling molecule, arteriole vasodilation can occur in the absence of astrocyte $\mathrm{Ca}^{2+}$ increases, $\mathrm{Ca}^{2+}$ signals are characterized by long latencies occurring after the initiation of vasodilation and $\mathrm{K}^{+}$siphoning through astrocytes does not always play a major role in neurovascular coupling. Moreover, hemodynamic modulatory pathways can have differing levels of influence across various structures. For instance, studies have shown that NO can be an active signaling molecule in the cerebellum (Akgoren et al., 1996; Yang and Iadecola 1997) and hippocampus (Lourenco et al., 2014).

A possible addition to this conventional approach are chloride channel-dependent mechanisms of neurovascular coupling, which may participate in neurovascular deficiency and neurodegeneration. Prominent pathways which employ such chloride channels are gamma aminobutyric acid (GABA) ergic interneuron pathways, which operate via GABA-gated chloride channels (GABA $A_{A}$ receptors) and provide a means of rapid signaling. The role of GABAergic interneurons and $\mathrm{GABA}_{\mathrm{A}}$ receptors in inhibition of neuronal activity is well-known. Interneurons suppress excessive neuronal activity and spatially limit neuronal responses by instigating the hyperpolarization of the cell membrane which has the added benefit of decreasing local oxygen consumption. Additionally, GABA-gated chloride channels can directly participate in regulating cerebral blood flow. $\mathrm{GABA}_{\mathrm{A}}$ receptors can be found along arterioles (Vaucher et al., 2000) where interneurons make direct morphological connections (Cauli et al., 2004; Tremblay et al., 2016). These chloride channels on brain vessels are functionally active and are able to facilitate substantial vasodilation in response to stimulation, attributable to the hyperpolarization of arteriolar smooth muscles with their subsequent relaxation. Multiple studies have shown that GABAergic interneurons are essential for the full expression of the hemodynamic response in the presence of chemical or electrical stimulation (Kocharyan et al., 2008), during epileptiform discharges (Saillet et al., 2016) as well as in response to both sensory (Aksenov et al., 2019) and optogenetic stimulation (Anenberg et al., 2015). Arteriolar GABA- 
gated chloride channels, can therefore play an important role in the hemodynamic response due to their fast and profound effect on vasodilation.

In essence, GABA-gated chloride channels can function to prevent hypoxia by both upregulating oxygen supply and downregulating oxygen consumption. Thus, it is our perspective that if the number of these channels or their main biochemical properties are affected, the combination of decreased inhibition and a weakened hemodynamic response can induce local hypoxia, which will alter the intracellular and extracellular environment with neurodegeneration evident thereafter. In support of this perspective, we will briefly review chloride channel dysfunction and neurodegeneration in different diseases, and then provide our interpretation regarding the role of neurovascular deficiency as a medium between chloride channel dysfunction and neurodegeneration.

\section{NEURODEGENERATION AND CHLORIDE CHANNEL DEFICIENCY}

Chloride channel deficiency accompanies many neurodegenerative diseases. For example, in Alzheimer's disease, which is characterized by progressive neurodegeneration starting in hippocampus and entorhinal cortex, the neurotransmission of GABA and GABAergic terminals have been shown to be significantly disrupted in areas neighboring beta-amyloid plaques (Li et al., 2016). Subsequent analysis has shown abnormal upregulation and downregulation of the $\alpha 2, \beta 1, \gamma 1$, and $\alpha 1, \gamma 2$ subunits of $\mathrm{GABA}_{\mathrm{A}}$ receptors respectively (Limon et al., 2012). Another example is Parkinson's disease. This progressive neurodegenerative disorder is strongly associated with neuronal cell loss in the substantia nigra and striatum (Fahn and Sulzer, 2004). Although Parkinson's disease mostly corresponds with the loss of dopaminergic neurons, GABA and $\mathrm{GABA}_{\mathrm{A}}$ receptor deficiency has also been shown to play an important role in the early and non-motor symptoms of Parkinson's disease (Murueta-Goyena et al., 2019). These changes in GABAergic pathways are different from those observed in Huntington's disease. In Huntington's disease GABAergic interneurons undergo specific morphological alterations (i.e., reduced somatic areas and dendritic field complexity) which accompanies aggressive neurodegeneration in the striatum (Bano et al., 2011).

The etiologies of Alzheimer's, Parkinson's and particularly Huntington's diseases, are often attributed to genetics, however, some diseases (for example, epilepsy) can be independent of such substantial genetic factors. Distinctly, Drug-Resistant Epilepsy (DRE), which occurs in $40 \%$ of people with epilepsy (Engel, 2016), has been shown to cause neurodegeneration, often in the temporal lobe. Evidence has elucidated the association between the increased internalization of $\mathrm{GABA}_{\mathrm{A}}$ receptors and symptoms in DRE (Goodkin et al., 2005; Naylor et al., 2005; Goodkin et al., 2007).

Even complex psychiatric disorders can present with chloride channel affiliated neurodegeneration. For instance, patients with schizophrenia exhibit progressive bilateral neurodegeneration in the grey matter of the temporal and parietal lobes (Whitford et al., 2006), and can exhibit significant under-expression of the $a 5$ subunit of $\mathrm{GABA}_{\mathrm{A}}$ receptors, the degree of which is correlated with the symptom severity (Marques et al., 2020). Furthermore, autism spectrum disorder (ASD) has demonstrated similar patterns of neurodegeneration to that of schizophrenia. Individuals with ASD have exhibited reduced grey matter volumes in the mirror neuron system (Hadjikhani et al., 2006; Marques et al., 2020). The severity of grey matter thinning in this area was further correlated with the severity of symptoms experienced by those with ASD. Moreover, genetic studies have identified copy number variations and entire locus duplications of the 15p11-q13 chromosomal region in patients with ASD, which lead to under and dysfunctional expression of the $\beta 3, \alpha 5$, and $\gamma 3$ subunits of $\mathrm{GABA}_{\mathrm{A}}$ receptors (Hadjikhani et al., 2006). This indicates the potential of chloride channel deficiency to both precede cases of ASD, and have further downstream consequences of neurodegeneration.

Chloride channel dysfunction and neurodegeneration can also occur as an acquired iatrogenic condition; the most notable example of which is neonatal exposure to anesthesia (Aksenov et al., 2020a). Anesthetics that are classified as GABA agonists and glutamate antagonists (Aksenov et al., 2019), have consistently been shown to produce significant neuroapoptosis that is directly correlated with dosage and duration of the anesthesia delivery (Hadjikhani et al., 2006; Zheng et al., 2015; Liu et al., 2018). Moreover, the severity of apoptosis can create a loss of cortical neurons, of which a significant proportion are GABAergic inhibitory interneurons (Istaphanous et al., 2013), and a further study has shown general anesthesia to directly disturb chloride channels (Cabrera et al., 2020) thereby broadening the known contributory effects of anesthesia on neurodegeneration (Aksenov, 2021). These neurodegenerative and apoptotic processes can alter the delicate excitatory/inhibitory balance of cortical networks (Aksenov et al., 2020a). This imbalance can account for, at least in part, the negative developmental changes (Johnston et al., 2002; Aksenov et al., 2020a; Aksenov et al., 2020b) and impeded GABAergic system development (Young et al., 2012; Nisimov et al., 2018) following neonatal anesthesia. This disproportionate cell death leading to a shift in the excitatory/inhibitory balance requires further research in terms of occurrence of the local chronic hypoxia in later years, and how this shift caused by anesthesia, adapts throughout development.

\section{DISCUSSION}

We suggest that, in the absence of normal $\mathrm{GABA}_{\mathrm{A}}$ receptor functioning, neurovascular deficiency could manifest where a weakened hemodynamic response, in combination with decreased inhibition, would be insufficient to support the present metabolic demand. Although this type of neurovascular deficiency does not result in actual ischemic stroke, it engenders chronic intermittent hypoxia which produces neurodegeneration. This clear sequence of events explains the importance of normal chloride channel 
functioning for preventing chronic hypoxia. Therefore, dysfunctional chloride channels could be a contributory factor to the neurodegeneration in the aforementioned diseases which are epiphenomenal with chloride channel dysfunction.

Indeed, the dangers of hypoxia on the intracellular and extracellular compositions of brain tissue have been well documented. It is known that insufficient oxygen for basic metabolic processes can lead to cell death (Mariotti et al., 2016). Although the neuronal damage is especially severe in sudden onset hypoxia-ischemia, such as in the case of an ischemic stroke, it can also occur as a result of chronic hypoxia (Dheer et al., 2018; Mahakizadeh et al., 2020). Depending on the severity, hypoxia has been shown to increase the production of reactive oxygen species which can accumulate beyond the protective abilities of anti-oxidative systems, causing oxidative stress (Chen et al., 2018). Oxidative stress has a high propensity to interact with macromolecules within cells (e.g., DNA/RNA oxidation, protein oxidation, nitration of tyrosine residues, and lipid peroxidation), leading to cell debilitation (Moreira et al., 2005). Other consequences of hypoxia include a reduction in intracellular and extracellular $\mathrm{pH}$ (Rolett et al., 2000; Yao and Haddad, 2004), phosphocreatine (Rolett et al., 2000), inorganic phosphate (Nioka et al., 1990; Rolett et al., 2000) and a buildup of NADH (Rolett et al., 2000; Shetty et al., 2014). These distinct alterations to the intracellular and extracellular environment significantly impair normal cellular functioning and have been shown to be biochemical indicators of neuroapoptosis. Such hypoxia-related events not only demonstrate the ability of insufficient cerebral blood flow to produce neurodegeneration in the immediate undersupplied tissues, but that it can also harmfully affect neighboring tissues as well.

Brain functioning and its metabolic support is a highly integrated process, and embedded within this complex system are GABAergic interneurons and the hemodynamic response. When neurodegeneration is present, determining if neurovascular deficiency precedes this process and exacerbates the neurodegeneration, or suffers as a direct consequence of an unbalanced excitatory/inhibitory system, remains a challenge. These two possibilities are accompanied by respective hypotheses and can therefore be examined by future studies in a controlled environment. A possibility of how one may address

\section{REFERENCES}

Akgören, N., Dalgaard, P., and Lauritzen, M. (1996). Cerebral Blood Flow Increases Evoked by Electrical Stimulation of Rat Cerebellar Cortex: Relation to Excitatory Synaptic Activity and Nitric Oxide Synthesis. Brain Res. 710 (12), 204-214. doi:10.1016/0006-8993(95)01354-7

Aksenov, D. P., Li, L., Miller, M. J., and Wyrwicz, A. M. (2016). Blood Oxygenation Level Dependent Signal and Neuronal Adaptation to Optogenetic and Sensory Stimulation in Somatosensory Cortex in Awake Animals. Eur. J. Neurosci. 44 (9), 2722-2729. doi:10.1111/ejn.13384

Aksenov, D. P., Li, L., Miller, M. J., and Wyrwicz, A. M. (2019). Role of the Inhibitory System in Shaping the BOLD fMRI Response. Neuroimage 201, 116034. doi:10.1016/j.neuroimage.2019.116034 this issue includes in vivo studies providing longitudinal measurement of chloride channel and interneuron deficiencies in association with subsequent hemodynamic function and neurodegeneration.

Further interrogation into chloride channel subunit functioning may provide a bottom-up approach to more accurately describe their role in neurodegeneration. A family of genes have been identified (regions CLC2-7) to transcript chloride channels in the brain (Jentsch et al., 1999). These loci represent specific areas of potential genetic manipulation that could identify the discrete contribution of chloride channels and their subunits in degenerative diseases. In addition, the local modulation of chloride channel expression with a viral vector could be used. This type of methodology has proven effective in animal translational models (Miah et al., 2019). Unfortunately, little work has been done to use viral vectors to modulate chloride channel expression in the brain. However, in reference to $\mathrm{GABA}_{\mathrm{A}}$ receptors, certain benzodiazepine derivatives have shown to allosterically bind to individual subunits. Namely, TPA023 (Atack et al., 2006), HZ166 (Di Lio et al., 2011) and SL651498 (Griebel et al., 2003) are reported to act as $\alpha 2$ and $\alpha 3$ agonists, while CGS 9865 binds to the $\beta+\alpha-$ interface (Maldifassi et al., 2016). Genetic and subunit-related research may provide further insights into chloride channel dysfunction and lead to etiologically-specific pharmacological solutions to both protect chloride channels, and prevent neurovascular deficiency, in the previously discussed diseases and conditions.

\section{AUTHOR CONTRIBUTIONS}

DA and DG wrote the manuscript, AD provided input on the manuscript. All authors contributed to manuscript revision, read, and approved the submitted version.

\section{FUNDING}

This work was supported by R01GM112715 (National Institute of General Medical Sciences), R01NS107383 (National Institute of Neurological Disorders and Stroke), R01NS119251 (National Institute of Neurological Disorders and Stroke).

Aksenov, D. P., Miller, M. J., Dixon, C. J., and Drobyshevsky, A. (2020a). Impact of Anesthesia Exposure in Early Development on Learning and Sensory Functions. Dev. Psychobiol 62 (5), 559-572. doi:10.1002/dev.21963

Aksenov, D. P., Miller, M. J., Dixon, C. J., and Wyrwicz, A. M. (2019). The Effect of Sevoflurane and Isoflurane Anesthesia on Single Unit and Local Field Potentials. Exp. Brain Res. 237 (6), 1521-1529. doi:10.1007/s00221-019-05528-9

Aksenov, D. P. (2021). Normal Development of Local Neurovascular Interactions and the Diagnostic Value of Resting State Functional MRI in Neurovascular Deficiency Based on the Example of Neonatal Anesthesia Exposure. Front. Neurol. 12, 664706. doi:10.3389/fneur.2021.664706

Aksenov, D. P., Venkatasubramanian, P. N., Miller, M. J., Dixon, C. J., Li, L., and Wyrwicz, A. M. (2020b). Effects of Neonatal Isoflurane Anesthesia Exposure on Learning-specific and Sensory Systems in Adults. Sci. Rep. 10 (1), 13832. doi:10.1038/s41598-020-70818-0 
Anenberg, E., Chan, A. W., Xie, Y., LeDue, J. M., and Murphy, T. H. (2015). Optogenetic Stimulation of GABA Neurons Can Decrease Local Neuronal Activity while Increasing Cortical Blood Flow. J. Cereb. Blood Flow Metab. 35 (10), 1579-1586. doi:10.1038/jcbfm.2015.140

Archer, S. L., Huang, J. M., Hampl, V., Nelson, D. P., Shultz, P. J., and Weir, E. K. (1994). Nitric Oxide and cGMP Cause Vasorelaxation by Activation of a Charybdotoxin-Sensitive K Channel by cGMP-dependent Protein Kinase. Proc. Natl. Acad. Sci. U S A. 91 (16), 7583-7587. doi:10.1073/pnas.91.16.7583

Atack, J. R., Wafford, K. A., Tye, S. J., Cook, S. M., Sohal, B., Pike, A., et al. (2006). TPA023 [7-(1,1-Dimethylethyl)-6-(2-Ethyl-2h-1,2,4-Triazol-3-Ylmethoxy)-3(2-Fluorophenyl)-1,2,4-Triazolo[4,3-B]pyridazine], an Agonist Selective for Alpha2- and Alpha3-Containing GABAA Receptors, Is a Nonsedating Anxiolytic in Rodents and Primates. J. Pharmacol. Exp. Ther. 316 (1), 410-422. doi:10.1124/jpet.105.089920

Attwell, D., Buchan, A. M., Charpak, S., Lauritzen, M., Macvicar, B. A., and Newman, E. A. (2010). Glial and Neuronal Control of Brain Blood Flow. Nature 468 (7321), 232-243. doi:10.1038/nature09613

Bano, D., Zanetti, F., Mende, Y., and Nicotera, P. (2011). Neurodegenerative Processes in Huntington's Disease. Cell Death Dis 2, e228. doi:10.1038/ cddis. 2011.112

Cabrera, O. H., Tesic, V., Tat, Q. L., Chastain, S., Quillinan, N., and JevtovicTodorovic, V. (2020). Sevoflurane-Induced Dysregulation of Cation-Chloride Cotransporters NKCC1 and KCC2 in Neonatal Mouse Brain. Mol. Neurobiol. 57 (1), 1-10. doi:10.1007/s12035-019-01751-1

Cauli, B., Tong, X. K., Rancillac, A., Serluca, N., Lambolez, B., Rossier, J., et al. (2004). Cortical GABA Interneurons in Neurovascular Coupling: Relays for Subcortical Vasoactive Pathways. J. Neurosci. 24 (41), 8940-8949. doi:10.1523/ JNEUROSCI.3065-04.2004

Chen, R., Lai, U. H., Zhu, L., Singh, A., Ahmed, M., and Forsyth, N. R. (2018). Reactive Oxygen Species Formation in the Brain at Different Oxygen Levels: The Role of Hypoxia Inducible Factors. Front Cel Dev Biol 6, 132. doi:10.3389/ fcell.2018.00132

Dheer, A., Jain, V., Kushwah, N., Kumar, R., Prasad, D., and Singh, S. B. (2018). Temporal and Spatial Changes in Glial Cells during Chronic Hypobaric Hypoxia: Role in Neurodegeneration. Neuroscience 383, 235-246. doi:10.1016/j.neuroscience.2018.04.026

Di Lio, A., Benke, D., Besson, M., Desmeules, J., Daali, Y., Wang, Z. J., et al. (2011). HZ166, a Novel GABAA Receptor Subtype-Selective Benzodiazepine Site Ligand, Is Antihyperalgesic in Mouse Models of Inflammatory and Neuropathic Pain. Neuropharmacology 60 (4), 626-632. doi:10.1016/ j.neuropharm.2010.11.026

Engel, J., Jr. (2016). What Can We Do for People with Drug-Resistant Epilepsy? the 2016 Wartenberg Lecture. Neurology 87 (23), 2483-2489. doi:10.1212/ WNL.0000000000003407

Fahn, S., and Sulzer, D. (2004). Neurodegeneration and Neuroprotection in Parkinson Disease. NeuroRx 1 (1), 139-154. doi:10.1602/neurorx.1.1.139

Goodkin, H. P., Sun, C., Yeh, J. L., Mangan, P. S., and Kapur, J. (2007). GABA(A) Receptor Internalization during Seizures. Epilepsia 48 (Suppl. 5), 109-113. doi:10.1111/j.1528-1167.2007.01297.x

Goodkin, H. P., Yeh, J. L., and Kapur, J. (2005). Status Epilepticus Increases the Intracellular Accumulation of GABAA Receptors. J. Neurosci. 25 (23), 5511-5520. doi:10.1523/JNEUROSCI.0900-05.2005

Griebel, G., Perrault, G., Simiand, J., Cohen, C., Granger, P., Depoortere, H., et al. (2003). SL651498, a GABAA Receptor Agonist with Subtype-Selective Efficacy, as a Potential Treatment for Generalized Anxiety Disorder and Muscle Spasms. CNS Drug Rev. 9 (1), 3-20. doi:10.1111/j.1527-3458.2003.tb00241.x

Hadjikhani, N., Joseph, R. M., Snyder, J., and Tager-Flusberg, H. (2006). Anatomical Differences in the Mirror Neuron System and Social Cognition Network in Autism. Cereb. Cortex 16 (9), 1276-1282. doi:10.1093/cercor/ bhj069

Istaphanous, G. K., Ward, C. G., Nan, X., Hughes, E. A., McCann, J. C., McAuliffe, J. J., et al. (2013). Characterization and Quantification of Isoflurane-Induced Developmental Apoptotic Cell Death in Mouse Cerebral Cortex. Anesth. Analg 116 (4), 845-854. doi:10.1213/ANE.0b013e318281e988

Jentsch, T. J., Friedrich, T., Schriever, A., and Yamada, H. (1999). The CLC Chloride Channel Family. Pflugers Arch. 437 (6), 783-795. doi:10.1007/ s004240050847
Johnston, M. V., Nakajima, W., and Hagberg, H. (2002). Mechanisms of Hypoxic Neurodegeneration in the Developing Brain. Neuroscientist 8 (3), 212-220. doi:10.1177/1073858402008003007

Kocharyan, A., Fernandes, P., Tong, X. K., Vaucher, E., and Hamel, E. (2008). Specific Subtypes of Cortical GABA Interneurons Contribute to the Neurovascular Coupling Response to Basal Forebrain Stimulation. J. Cereb. Blood Flow Metab. 28 (2), 221-231. doi:10.1038/sj.jcbfm.9600558

Li, Y., Sun, H., Chen, Z., Xu, H., Bu, G., and Zheng, H. (2016). Implications of GABAergic Neurotransmission in Alzheimer's Disease. Front. Aging Neurosci. 8, 31. doi:10.3389/fnagi.2016.00031

Limon, A., Reyes-Ruiz, J. M., and Miledi, R. (2012). Loss of Functional GABA(A) Receptors in the Alzheimer Diseased Brain. Proc. Natl. Acad. Sci. U S A. 109 (25), 10071-10076. doi:10.1073/pnas.1204606109

Liu, Y., Liu, C., Zeng, M., Han, X., Zhang, K., Fu, Y., et al. (2018). Influence of Sevoflurane Exposure on Mitogen-Activated Protein Kinases and Akt/GSK-3 $\beta$ / CRMP-2 Signaling Pathways in the Developing Rat Brain. Exp. Ther. Med. 15 (2), 2066-2073. doi:10.3892/etm.2017.5651

Lourenço, C. F., Santos, R. M., Barbosa, R. M., Cadenas, E., Radi, R., and Laranjinha, J. (2014). Neurovascular Coupling in hippocampus Is Mediated via Diffusion by Neuronal-Derived Nitric Oxide. Free Radic. Biol. Med. 73, 421-429. doi:10.1016/j.freeradbiomed.2014.05.021

Mahakizadeh, S., Mokhtari, T., Navaee, F., Poorhassan, M., Tajik, A., and Hassanzadeh, G. (2020). Effects of Chronic Hypoxia on the Expression of seladin-1/Tuj1 and the Number of Dark Neurons of hippocampus. J. Chem. Neuroanat. 104, 101744. doi:10.1016/j.jchemneu.2020.101744

Maldifassi, M. C., Baur, R., and Sigel, E. (2016). Molecular Mode of Action of CGS 9895 at $\alpha 1 \beta 2 \gamma 2$ GABAAreceptors. J. Neurochem. 138 (5), 722-730. doi:10.1111/ jnc. 13711

Mariotti, L., Losi, G., Sessolo, M., Marcon, I., and Carmignoto, G. (2016). The Inhibitory Neurotransmitter GABA Evokes Long-Lasting $\mathrm{Ca}(2+)$ Oscillations in Cortical Astrocytes. Glia 64 (3), 363-373. doi:10.1002/glia.22933

Marques, T. R., Ashok, A. H., Angelescu, I., Borgan, F., Myers, J., Lingford-Hughes, A., et al. (2020). GABA-A Receptor Differences in Schizophrenia: a Positron Emission Tomography Study Using [(11)C]Ro154513. Mol. Psychiatry.

Miah, K. M., Hyde, S. C., and Gill, D. R. (2019). Emerging Gene Therapies for Cystic Fibrosis. Expert Rev. Respir. Med. 13 (8), 709-725. doi:10.1080/ 17476348.2019.1634547

Moreira, P. I., Smith, M. A., Zhu, X., Nunomura, A., Castellani, R. J., and Perry, G. (2005). Oxidative Stress and Neurodegeneration. Ann. N. Y Acad. Sci. 1043, 545-552. doi:10.1196/annals.1333.062

Murueta-Goyena, A., Andikoetxea, A., Gómez-Esteban, J. C., and Gabilondo, I. (2019). Contribution of the GABAergic System to Non-motor Manifestations in Premotor and Early Stages of Parkinson's Disease. Front. Pharmacol. 10, 1294. doi:10.3389/fphar.2019.01294

Naylor, D. E., Liu, H., and Wasterlain, C. G. (2005). Trafficking of GABA(A) Receptors, Loss of Inhibition, and a Mechanism for Pharmacoresistance in Status Epilepticus. J. Neurosci. 25 (34), 7724-7733. doi:10.1523/ JNEUROSCI.4944-04.2005

Nioka, S., Chance, B., Smith, D. S., Mayevsky, A., Reilly, M. P., Alter, C., et al. (1990). Cerebral Energy Metabolism and Oxygen State during Hypoxia in Neonate and Adult Dogs. Pediatr. Res. 28 (1), 54-62. doi:10.1203/00006450199007000-00013

Nippert, A. R., Biesecker, K. R., and Newman, E. A. (2018). Mechanisms Mediating Functional Hyperemia in the Brain. Neuroscientist 24 (1), 73-83. doi:10.1177/ 1073858417703033

Nisimov, H., Orenbuch, A., Pleasure, S. J., and Golan, H. M. (2018). Impaired Organization of GABAergic Neurons Following Prenatal Hypoxia. Neuroscience 384, 300-313. doi:10.1016/j.neuroscience.2018.05.021

Rolett, E. L., Azzawi, A., Liu, K. J., Yongbi, M. N., Swartz, H. M., and Dunn, J. F. (2000). Critical Oxygen Tension in Rat Brain: a Combined (31)P-NMR and EPR Oximetry Study. Am. J. Physiol. Regul. Integr. Comp. Physiol. 279 (1), R9-R16. doi:10.1152/ajpregu.2000.279.1.R9

Ross, W. N., and Manita, S. (2012). Imaging Calcium Waves and sparks in central Neurons. Cold Spring Harb Protoc. 2012 (3), 1087-1091. doi:10.1101/ pdb.prot071480

Saillet, S., Quilichini, P. P., Ghestem, A., Giusiano, B., Ivanov, A. I., Hitziger, S., et al. (2016). Interneurons Contribute to the Hemodynamic/metabolic 
Response to Epileptiform Discharges. J. Neurophysiol. 115 (3), 1157-1169. doi:10.1152/jn.00994.2014

Shetty, P. K., Galeffi, F., and Turner, D. A. (2014). Nicotinamide Pre-treatment Ameliorates $\mathrm{NAD}(\mathrm{H})$ Hyperoxidation and Improves Neuronal Function after Severe Hypoxia. Neurobiol. Dis. 62, 469-478. doi:10.1016/j.nbd.2013.10.025

Tremblay, R., Lee, S., and Rudy, B. (2016). GABAergic Interneurons in the Neocortex: From Cellular Properties to Circuits. Neuron 91 (2), 260-292. doi:10.1016/j.neuron.2016.06.033

Vaucher, E., Tong, X. K., Cholet, N., Lantin, S., and Hamel, E. (2000). GABA Neurons Provide a Rich Input to Microvessels but Not Nitric Oxide Neurons in the Rat Cerebral Cortex: a Means for Direct Regulation of Local Cerebral Blood Flow. J. Comp. Neurol. 421, 161-171. doi:10.1002/(sici)1096-9861(20000529) 421:2<161::aid-cne3>3.0.co;2-f

Whitford, T. J., Grieve, S. M., Farrow, T. F., Gomes, L., Brennan, J., Harris, A. W., et al. (2006). Progressive Grey Matter Atrophy over the First 2-3 Years of Illness in First-Episode Schizophrenia: a Tensor-Based Morphometry Study. Neuroimage 32 (2), 511-519. doi:10.1016/j.neuroimage.2006.03.041

Yang, G., and Iadecola, C. (1997). Obligatory Role of NO in Glutamate-dependent Hyperemia Evoked from Cerebellar Parallel Fibers. Am. J. Physiol. 272 (4 Pt 2), R1155-R1161. doi:10.1152/ajpregu.1997.272.4.R1155

Yao, H., and Haddad, G. G. (2004). Calcium and pH Homeostasis in Neurons during Hypoxia and Ischemia. Cell Calcium 36 (3-4), 247-255. doi:10.1016/ j.ceca.2004.02.013

Young, S. Z., Taylor, M. M., Wu, S., Ikeda-Matsuo, Y., Kubera, C., and Bordey, A. (2012). NKCC1 Knockdown Decreases Neuron Production through
GABA(A)-regulated Neural Progenitor Proliferation and Delays Dendrite Development. J. Neurosci. 32 (39), 13630-13638. doi:10.1523/ JNEUROSCI.2864-12.2012

Zheng, S. Q., Chen, X., Wang, Y. J., and An, L. X. (2015). [Effects of Sevoflurane on Brain Neuroapoptosis and Ability of Long-Term Learning and Memory in Newborn Rats]. Beijing Da Xue Xue Bao Yi Xue Ban 47 (4), 674-678.

Conflict of Interest: The authors declare that the research was conducted in the absence of any commercial or financial relationships that could be construed as a potential conflict of interest.

Publisher's Note: All claims expressed in this article are solely those of the authors and do not necessarily represent those of their affiliated organizations, or those of the publisher, the editors and the reviewers. Any product that may be evaluated in this article, or claim that may be made by its manufacturer, is not guaranteed or endorsed by the publisher.

Copyright (๑) 2021 Gascoigne, Drobyshevsky and Aksenov. This is an open-access article distributed under the terms of the Creative Commons Attribution License (CC $B Y$ ). The use, distribution or reproduction in other forums is permitted, provided the original author(s) and the copyright owner(s) are credited and that the original publication in this journal is cited, in accordance with accepted academic practice. No use, distribution or reproduction is permitted which does not comply with these terms. 